\title{
Concentraciones plasmáticas normales de magnesio y calcio en madres y recién nacidos
}

\author{
Dra. Andrea Gleisner E., 1 ; Q.F. Carlos Bruhn F.2; Dr. Guillemo Venegas V.1; \\ Dra. Margot Jonas O.; Dr. Raúl Higueras F,3; Prof. Elena Jarpa F.4 \\ Plasma levels of magnesium and calcium in healthy \\ mothers and their newborn infants
}

\begin{abstract}
Plasma levels of magnesium and caicium were meusured in 22 healthy mothers at the time of delivery and in their newborn infants. Plasma levels of magnesium in newborns (mean 2.50 0.35 S.D. mg $x$ di) were significantly higher ( $p<0.01$ ) than cord's (mean $1.87 \pm 0.18 \mathrm{~S} . \mathrm{D}$. mg $x \mathrm{dl}$ ) and mothers' blood levels (mean $1.78 \pm 0.23 \mathrm{~S} . \mathrm{D} . \mathrm{mg}$ $x$ dlj. Calcium plasma levals in mothers (mean $10.56 \pm 0.67$ S.D. mg $x$ dl) were significantly lower than those of

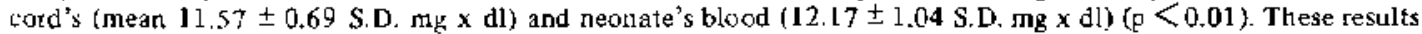
are similar to those obtained by foreign authors. There was no direct correlation between plasma levels of magnesium and calcium.

(Key words: magnesium. calcium, blood levels, maternal, newborn infants, cord blood).
\end{abstract}

Los recién nacidos (KN) provienen de un medio (intrauterino) donde glucosa: calcio (Ca) y magnesio $(\mathrm{Mg})$ son aportados continuamente $y$ sus concentraciones plasmáticas (fetales) son estrechamente reguladas por la homeostasis materna y el intercambio placentario. La suspensión brusca de este aporte al nacer demanda cambios

1. Departamento de Pediatría, Facultad de Medicina, Universidad de Concepción.

2. Departamento de Análisis Instrumental, Facultad de Farmacia. Universidad de Conicepción.

3. Servicio de Pediatría, Hospital Guillermo Grant Benavente, Concepción.

4. Departamento de Estadística, Facultad de Ciencias. Universidad de Concepción.

Esta investigación fue financiada por la Disección de Investigación, Liniversidad de Concepción, Proyecto 21.08.01. profundos en el metabolismo energético y mineral. que dependen de mecanismos reguladores que pueden estar aún inmaduros.

La placenta transporta activamente $\mathrm{Ca}$ y $\mathrm{Mg}$ hacia el feto. ${ }^{1,2}$ El transporte activo de $\mathrm{Ca}$ hacia el feto mantiene concentraciones plasmáticas fetales superiores a la calcemia materna, permitiendo la calcificación fetal normal. ${ }^{2}$ El transporte de $\mathrm{Mg}$, a diferencia del $\mathrm{Ca}$, es afectado adversamente por la insuficiencia placentaria y la hipomagnesemia materna: la mitad del $\mathrm{Mg}$ corporal total (en contraste con $1 \%$ del $\mathrm{Ca}$ ) se encuentra en los tejidos blandos y el plasma. La hipomagnesemia es, por lo tanto, un reflejo de escasez verdadera de Mg en el RN y no de una alteración en la homeostasis ósea. ${ }^{1}$

La hipermagnesemia secundaria al tratamiento de la toxemia materna con sulfato de magnesio causa, por atra parte, serios trastomos en el 
neonato como hipotonia, depresión respiratoria y motilidad gastrointestinal disminuida. 2,3,4

Los síntomas de la hipomagnesemia e hipocalcemia son similares $y$, con frecuencia, ambos trastornos metabólicos coexisten. Los RN afectados suelen tener antecedentes comunes, como diabetes materna, hipoparatiroidismo, exangu1neo transfusión, mala absorción, o exceso de fósforo en la dieta. ${ }^{1,6}$ El déficit de Mg disminuye la liberación de hormona paratíroidea y también reduce el efecto de ésta sobre el metabolismo del $\mathrm{Ca}^{5}$

Estudios en neonatos de término y pretérmino demuestran las interrelaciones entre estos minerales $y$ otros como fósforo, zinc y cobre $;^{7,9}$ los efectos de la alimentación materna y de distintas fórmulas lácteas sobre los respectivos balan$\operatorname{ces}^{7-12}$ y la acción de otras sustencias como glucosa, vitamina $D$, hormona paratiroidea $y$ calcitonina, sobre la homeostasis de ambos. ${ }^{2}$. $13-16$

Este estudio se realizó con el propósito de describir las concentraciones normales de $\mathrm{Mg}$ en madres $y$ sus neonatos sanos, y establecer su relación con los valores normales de Ca. Con ello pretendemos contribuir, a nịvel regional, a una mejor interpretación de las mediciones sistemáticas de $\mathrm{Mg}$ en $\mathrm{RN}$ con trastornos metabólicos.

\section{MATERIAL Y METODO}

En la maternidad del Hospital Guillermo Grant Benavintc se tomaron muestras para determinar Jos niveles plasmáticos de $\mathrm{Mg}$ y $\mathrm{Ca}$ en 22 madres $y$ sus respectivos RN. Las madtes cumpluan los siguientes requisitos: edad entre 18 y 35 años, ausencia de enfermedades en el embarazo y parto raginal sin complieaciones. Los RN debían ser de térnino, de peso adecuado a su edad de gestación y calificados por el neonatólogo como normales. Las muestras de sangre materna sc obtuvieron durante el parto; las de los RN simultáneamente del cordón y del talón (muest ta capilar). Antes de transcurridos 30 minutos desde su extracción fueton centrifugadas, Iefrigeradas y analizadas por duplicato mediante un espectrofotómetro modelo Perkin Elmer 308. El estudio estadístico se efectuó aplicando análisis de varianza, pruebu " $t$ " y diagrama de dispersión.

\section{RESULTADOS}

Las concentraciones plasmáticas de $\mathrm{Mg}$ en las madres variaron entre 1,40 y $2,24 \mathrm{mg} x \mathrm{dl}$, promedio $(\bar{x}) 1,78 \mathrm{D} . \mathrm{E} . \pm 2.23 \mathrm{mg} \mathrm{x}$ dl. No hubo diferencia significativa $(p>0,05)$ con los niveles de Mg en el cordón ( $\bar{x} 1,87 \pm 0,18 \mathrm{mg} x$ dl, rango 1,52 a $2,17 \mathrm{mg} x \mathrm{dl}$ ). Se encontraron diferencias significativas $(p<0,01)$ entre los valores anteriores y los de sangre capilar de los $\mathrm{RN}(\overline{\mathrm{x}} 2,50 \pm$ $0,35 \mathrm{mg} x \mathrm{~d}$ ?, rango 1,95 a $3,35 \mathrm{mg} x \mathrm{dl}$ ) (figura 1 ).

Las calcemias maternas fluctuaron entre 9,27 y $12,24 \mathrm{mg} x$ dl $(\bar{x} 10,56 \pm 0,67 \mathrm{mg} \mathrm{x} \mathrm{dl})$, valores significativamente diferentes $(p<0,01)$ con los del cordón $(\bar{x}]$ I $, 57 \pm 0,69 \mathrm{mg} x \mathrm{dl}$, rango 10,61 a $12,96 \mathrm{mg} \mathrm{x}$ dl) y la sangre capilar de los $\mathrm{RN}(\overline{\mathrm{x}}$ $12,17 \pm 1,04 \mathrm{mg} x \mathrm{dl}$, rango 10,06 a $13,76 \mathrm{mg} x$ di) (figura 2)

En el diagrama de dispersión no encontramos

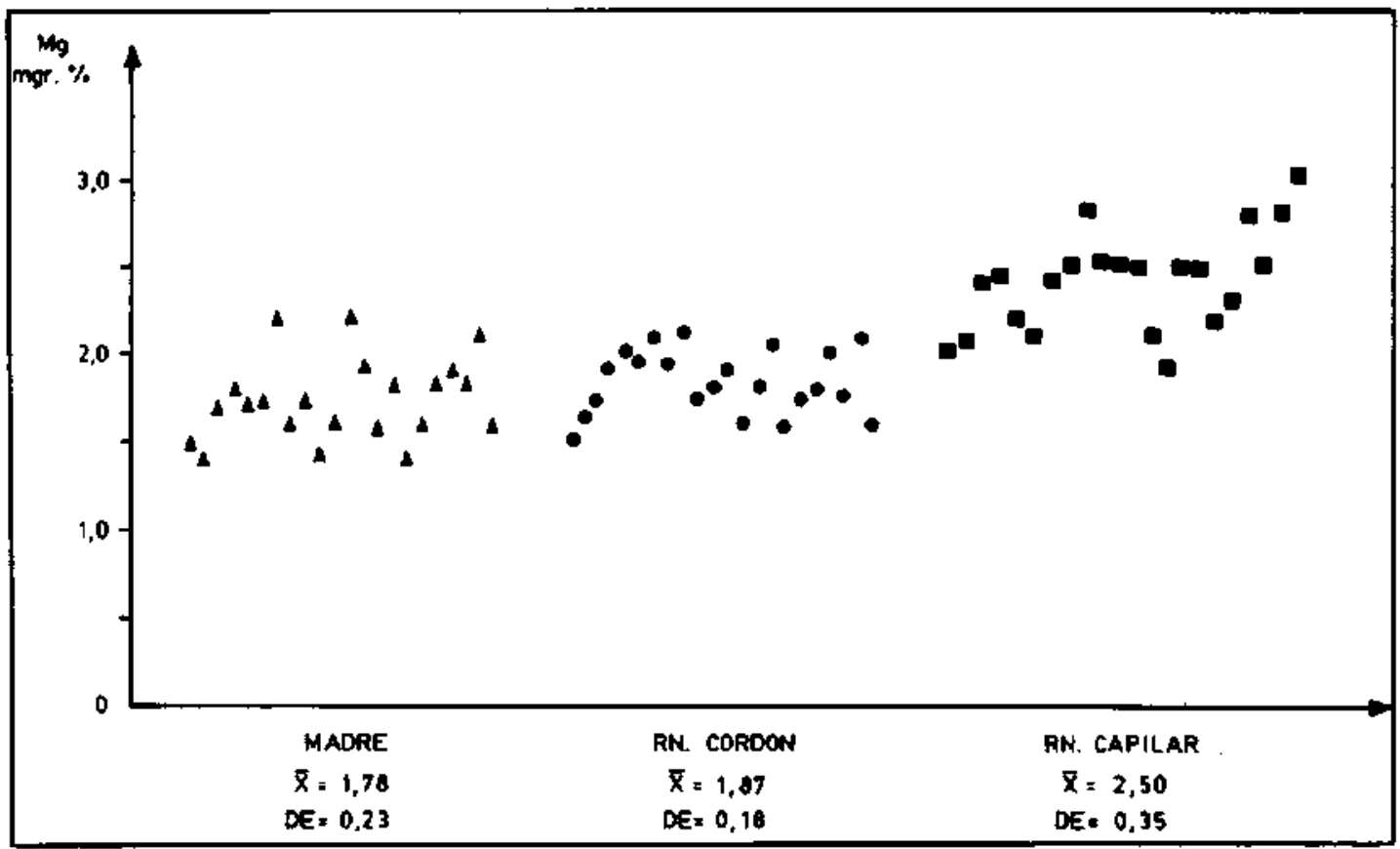

Figura 1 : Concentraciones normales de magnesio plastnático (mu $\mathrm{x}$ di). en madres y sus rectén nacjdos. (A madre cordón $\mathbf{\Delta a r g r e ~ c a p i l a r ~ d e ~ r e c i e ́ n ~ n a c i d o s ) . ~}$ 


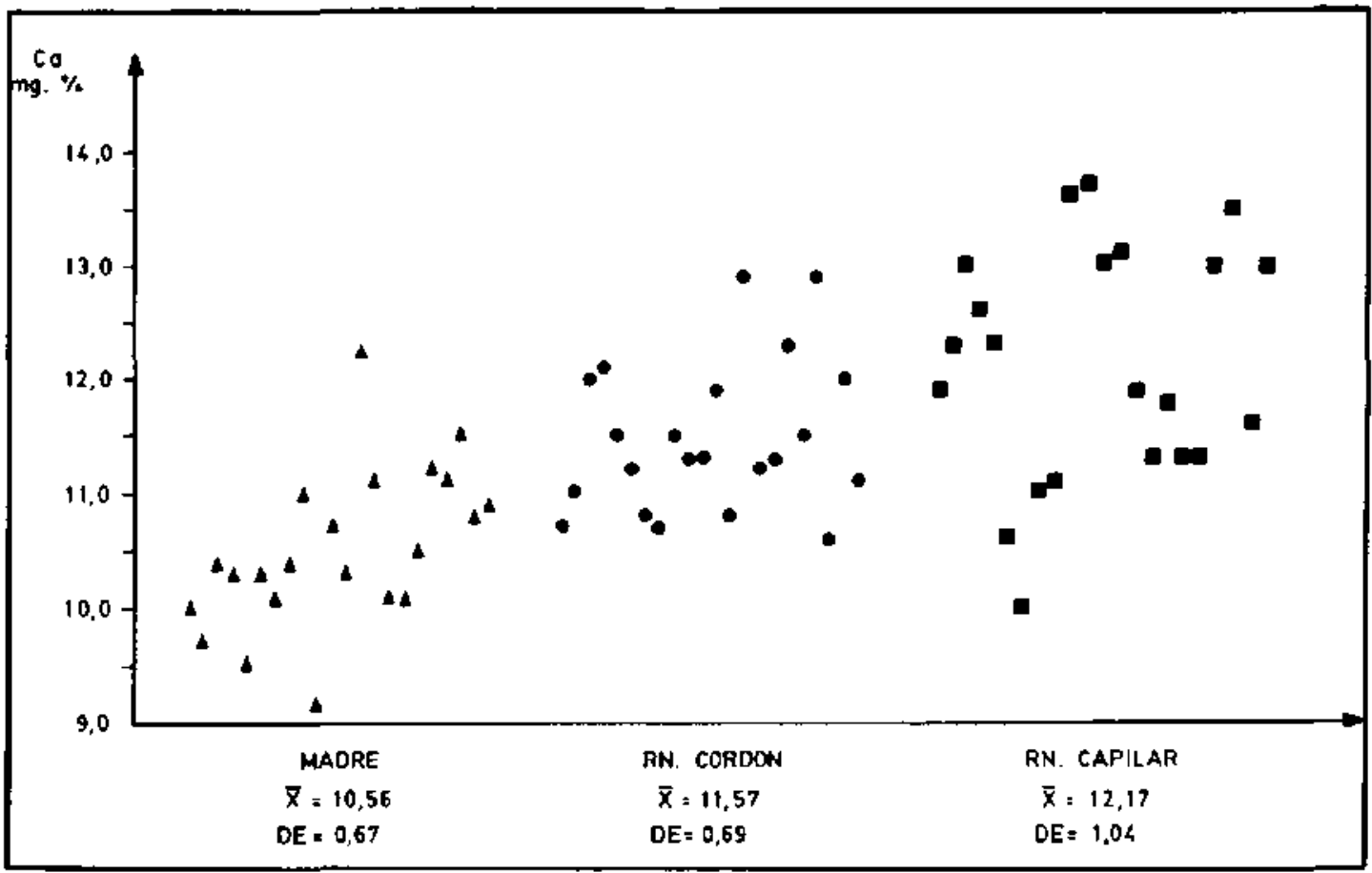

Figura 2: Concentraciones notmales de calcio plasmático (mg $\mathrm{x}$ dl), en madres y sus recién nacidos. ( $\boldsymbol{\Lambda}_{\text {madre }}$ cordón $\mathbf{\omega}$ sangre capilar de recién nacidos).

correlaciones lineales entre las concentraciones de Ca y Mg en las madres $(r=0,38)$ ni en los $R N$ ( $r=0,29$ y $r=0,04)$.

\section{COMENTARIO}

La concentración plasmática de $\mathrm{Mg}$ y $\mathrm{Ca}$ en madres sanas y sus RN normales, están dentro del rango señalado por diferentes autores, extranje$\operatorname{ros}^{1,16,17}$ y nacionales ${ }^{18}$.

Los valores de $\mathrm{Mg}$ en la sangre capilar de los RN fueron significativamente mayores que los maternos ( $\mathrm{p}<0,01)$, diferencia que fue aún más significativa $(\mathrm{p}<0,001)$ al comparar las calcemias entre neonatos y madres, tal había sido descrito por otros. $1,9.16,17$

- No se han encontrado relaciones entre los valores plasmaticos de $\mathrm{Mg}$ y $\mathrm{Ca}$ en el periodo neonatal durante las primeras $48 \mathrm{~h}$ de vida, ${ }^{19}$ lo que estaría de acuerdo con nuestros resultados. Sin embargo dicha correlación se establecería al octavo día de vida. ${ }^{9}$

El presente estudio demostró, además, el valor del micrométodo como técnica simple y factible de utilizar en nuestro medio para determinar los niveles plasmáticos de $\mathrm{Mg}$ y $\mathrm{Ca}$. Esto es de especial importancia en el periodo neonatal, donde se requieren procedimientos diagnósticos frecuentes para la identificación oportuna de cualquier cambio metabólico.

\section{RESUMEN}

Se determinaron los niveles plasmáticos de $\mathrm{Mg}$ y Ca en 22 madres y sus recién nacidos (RN). La concentración plasmática de $\mathrm{Mg}$ en las madres fue en promedio $(\bar{x})=1,78 \pm 0,23 \mathrm{mg} x \mathrm{~d} 1$; el valor en el cordón del $R N$ : $\bar{x}=1.87 \pm 0,18 \mathrm{mg} x$ di y en el capilar del $\mathrm{RN}: \overline{\mathrm{x}}=2,50 \pm 0,18 \mathrm{mg} \mathrm{x}$ dl. Este ultimo valor fue significativamente mayor que los anteriores $(p<0,01)$. Las calcemias maternas obtenidas fueron en $x=10,56 \pm 0,67 \mathrm{mg} \mathrm{x} \mathrm{dl}$, valores significativamente menores $(p<0,01)$ que los valores del cordón del $\mathrm{RN}: \overline{\mathrm{x}}=11,57 \pm$ $0,69 \mathrm{mg} x$ dl y del capilar $R N: \vec{x}=12,17 \pm 1,04 \mathrm{mg}$ $x$ dl. No encontramos correlación entre los valores de $\mathrm{Ca}$ y $\mathrm{Mg}$ en madres ni RN. Los valores de $\mathrm{Ca}$ y $\mathrm{Mg}$ obtenidos están dentro de los descritos por la literatura. Se demostró el valor del micrométodo como técnica útil en el período neoriatal para determinar los niveles plasmáticos de $\mathrm{Mg}$ y $\mathrm{Ca}$.

\section{REFERENCIAS}

1. Kliegmann R, Wald M.: Problems in Metabolic Adaptation: glucose, calcium, and magnesium. En: K!aus and Fanaroff: Care of the high-risk neonate. 3rd ed. Philadelphia, W., B. Saunders Company, 1986 .

2. Donovan E., Tsang R., Steichen Y., Strub R., Chen W. M.: Veonatal hypermagnesemia: Effect on parathyrold hormone and calcium homeostasis. J Pediatr 1980, 96:105-310. 
3. Wilkins I., Goldberg Y., Phillips R., Bacall Ch., Chervenack $F$., Berkowitz $R$.: Long-tern use of the magnesium sulfate as a tocolytic aqent. Obstet Gy necol 1986; 67: 385-405.

4. Mc Guinness $G$, Weinstein M., Cruikshank D., et al: Effect of magnesium sulfate treatment on perinatal calcium metabolism. Il Neonatal responses. Obstet Gy necol 1980; 56: 595-598.

5. Root $A$.. and Hartison H.: Recent avances in calcium metabolism. J Pediatr 1976; 88: 1-5.

6. Cockburn $F_{1}$, Brown $J$, Belton $N_{1}$, er al: Neonatal convulsions associated with a primary disturbance of calcium, phosphorus, and magnesium metabolism. Arch Dis Child 1973;48: 99-103.

7. Dauncey M., Shaw J., Urman J.: The absorption and tetention of magnesium, zinc and copper by low birth weight infants fed pasteurized human breast miłk. Pediat Res 1977; 11: $991-997$.

8. Schanler R., Garza C., O'Brian Smith E.: Fortified mothers' milk for very low birth weight intants: Results of mineral bajance studies. J Pediatr 1985: 107: 767-774.

9. Snodgrass G., Stimmler L., Went J., Ahrams M., Will $E$.. Interrelations of plasma calcium, inorganic phosphate, magnesium, and protein over the first week of like. Arch Dis Child 1973; 48: 279-285.

10. Tantibhedhyangkul $P$. Hashim $S_{\text {. }}$ : Medium chain triglyceridc feeding in premature infants: Effects on calcium and nagnesium absorption. Pediatrics $1978 ; 61: 537-545$

11. White R., Campbell D., Stanhope R., Bayley H., Sinclair $J$.: Fnergy balance in low birth wcight infunts fed fortnula of high or low medium - chain triglyceride content. J Pediats 1986; 108: 964-971.

12. Cooke R. Nichoalds G.: Nutuent retention in preterm infants fed standard infant formulas. J Pediatr 1986 ; 108 : $448-451$.

13."Venkataraman P., Blick K., Rao R., Fry H., Parker $M$. : Decline in sorum calcium, magnesium, and phosphorus values with oral glucose in normal neonates: Studies of serum parathyroid hormone and calcitontn. J Pediatr 1986; 108: 607-610.

14. Salle B., David $L$, Chopard $J$., Grafmeyer $D$., Renaud $H$. : Prevention of early neonatal hypocal. cemia in low birth weigth infants with continuous calcium infusion: Effect on serum calcium, phosphorus, magnesium and circulating inmuno. reactive parathyroid hormone and calcitonin. Pediatr Res $1977 ; 11 ; 1180-1185$.

15. Rude R, Adams J, Ryzen E. et al: Low serum concentrations of 1,25 dihidroxyritamin $D$ in human magnesium deficiency. J Clin Endocrinol Metab $1985 ; 61: 933-937$.

16. Reitz $R$., Dadne T., Woods J., Weinstein R.: Calcium, magnesium, phosphorus, and parathyroid hormone intcrelationships in pregnancy and newborn infants. Obstet Gynecol 1977; 50: 701.705 .

17. Behrman R., Klieginan R.: El feto y el recién nacido. En: Nelson W., Behrman R., Vaughan V.: Tratado de Pediatría 9a ed. Interamericana México, 1985,

18. Gleisner A., Soto G., Venegas G., Solar O., Rodriguez $A_{\text {.: }}$ Concentraciones plasmáticas de magnesio en lactantes con diarrea aguda, deshidratación $y$ acjdosis. Rey Chî Pediatr 1985; 56: 454-456.

19. Venkataraman P. Tsang R., Steichen J., Grey I., Neylan $M$. Fleischman $A_{\text {.: }}$ Early neonatal hy pocalcemia in extremely preterm in infants. AJDC 1986; 140: $1004 \cdot 1008$. 